\title{
Inappropriate Antibiotic Allergy Documentation in Health Records: A Qualitative Study on Family Physicians' and Pharmacists' Experiences
}

Kitty De Clercd, MD, MSc

Jochen W.L. Cals, MD, PbD

Eefje G.P.M. de Bont, MD, PbD

Department of Family Medicine, CAPHRI Care and Public Health Research Institute, Maastricht University Medical Centre, The Netherlands

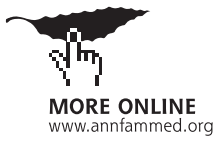

AC Annals Journal Club selection; see inside back cover or www.AnnFamMed. org/content/AJC/.

Conflict of interest: authors report none.

\section{CORRESPONDING AUTHOR}

Eefje G.P.M. de Bont, MD, PhD

Maastricht University

Department of Family Medicine

PO Box 616

6200 MD Maastricht, The Netherlands

eefje.debont@maastrichtuniversity.nl

\begin{abstract}
PURPOSE It is hypothesized that $90 \%$ of antibiotic allergies documented in patients' health records are not actual, potentially life threatening, type I allergies mediated by IgE. This distinction is important because such documentation increases antibiotic resistance, as more second-choice and broad-spectrum antibiotics are then used. Evidence is lacking regarding causes of this inappropriate documentation. To develop interventions aimed at improving documentation, we explored experiences of family physicians and pharmacists in this area.
\end{abstract}

METHODS We conducted a qualitative study among family physicians and pharmacists using focus group discussions, based on purposeful sampling and a naturalistic approach. Discussions were audio-recorded, transcribed verbatim, and analyzed in duplicate by means of constant comparative technique.

RESULTS We conducted 4 focus group discussions among 34 family physicians and 10 pharmacists, from which 3 main themes emerged: (1) magnitude and awareness of the problem of inappropriate antibiotic allergy documentation, (2) origin of the problem, and (3) approaches for addressing the problem. Participants noted that the magnitude of contamination of medical files with inappropriate documentation leads to skepticism about current documentation. Major hindering factors are electronic health record systems and electronic communication. In addition, family physicians and pharmacists believed they had insufficient knowledge about antibiotic allergies and called for tools to rectify inappropriate allergy documentation and facilitate proper documentation going forward.

CONCLUSIONS Family physicians and pharmacists perceive that few documented antibiotic allergies are in fact correct. Electronic health record barriers and communication barriers, as well as a lack of knowledge and facilitating tools, are main causes for numerous inappropriately documented antibiotic allergies and therefore targets for improving documentation in the future.

Ann Fam Med 2020;18:326-333. https://doi.org/10.1370/afm.2537.

\section{INTRODUCTION}

A ntibiotics are one of the most commonly prescribed types of medication because they are effective against common bacterial infections ${ }^{1-4}$; however, antibiotic resistance is an increasing threat to global health. An important factor contributing to antibiotic resistance in western countries is inappropriate documentation of antibiotic allergies in patients' medical records. ${ }^{5,6}$ Additionally, the high number of inappropriate documentations reduces antibacterial treatment options, as presence of a documented allergy precludes use of those antibiotics to which the patient is thought to be allergic. ${ }^{1-3}$ It is hypothesized that up to $90 \%$ of documented antibiotic allergies are not real, potentially life threatening, type I allergies mediated by $\operatorname{IgE} .{ }^{1,7}$

Avoiding certain groups of antibiotics because of inappropriate documentation of antibiotic allergies has negative consequences. First, the patient does not receive the optimal treatment, and alternative antibiotics 
can lead to more adverse reactions or superinfections. These factors eventually result in longer treatment duration, more complications, and worse

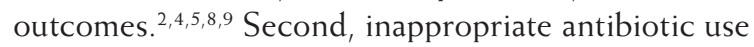
reinforces development of antimicrobial resistance, as the alternative antibiotics generally have a broader spectrum of activity, resulting in more deadly bacterial infections. ${ }^{2,4,10}$ Consequently, this situation increases health care costs because of the greater number of antibiotic prescriptions, longer time on therapy, and extended duration of hospitalization. ${ }^{4,9,11}$

Documentation of antibiotic allergies is mainly performed in primary care by family physicians, pharmacists, and their assistants. ${ }^{11}$ Although it is known that there are numerous inappropriate antibiotic allergy documentations, evidence is lacking regarding the experiences of family physicians and pharmacists performing and working with these documentations. To develop interventions for accurate documentation in the future, it is necessary to gain better insight into these experiences and to identify the causes of inappropriate documentation. This qualitative study therefore aimed to explore the experiences of family physicians and pharmacists performing and encountering antibiotic allergy documentations. We hypothesized that by identifying the main determinants leading to inappropriate documentation, using a qualitative approach, we can develop interventions for accurate documentation going forward.

\section{METHODS}

\section{Study Design}

We conducted a qualitative study among family physicians and pharmacists using focus group discussions based on a naturalistic approach. ${ }^{12}$ This approach allowed us to examine how inappropriate documentation happens and is experienced in actual daily practice. Participants were questioned about their experiences and expectations regarding the documentation of antibiotic allergies. Focus group discussions were chosen because they are an efficient way of collecting qualitative data from varied perspectives, and the group interaction provides more insight on the topic. ${ }^{13-15}$

\section{Setting}

The focus group discussions were carried out among family physicians and pharmacists from different cooperatives in their routine pharmacotherapeutic audit meetings in South Limburg, the Netherlands. Family physicians in the country are organized into cooperatives (groups), in which they collaborate to organize acute care in the area, establish local working arrangements, and plan regular and structured education sessions with local pharmacists, called pharmacotherapeutic audit meetings. Family physicians and pharmacists worked in the same region, sometimes in the same building, but pharmacies were separate entities.

\section{Participants}

Using purposeful sampling, we approached existing cooperatives of family physicians and pharmacists in the area by e-mail with a request to participate in the focus group discussions. By selecting groups based on different family physician and pharmacy information systems (the computer software they used for patients' medical records), varied composition of the cooperatives (region, number of family physicians belonging to the cooperative), diverse backgrounds of the participants (academic vs nonacademic family physicians, pharmacists from different pharmacies, varied age and number of years of experience), we expected to gain a diverse insight into the possible approaches to documentation, the communication regarding documentation within and between family physicians and pharmacists, and the causes of inappropriate documentation in this setting. Each focus group had a minimum of 4 participants, including at least 1 pharmacist. The number of focus groups was determined by data saturation.

\section{Data Collection}

We used sensitizing concepts ${ }^{16}$ to derive a topic list regarding the documentation of antibiotic allergies in general practices and pharmacies. On the basis of the literature and a priori exploratory interviews with 3 family physicians and 3 pharmacists, we prepared questions and compiled a list of topics: the responsibility and performance of documentation, the prevalence of inappropriate antibiotic allergy documentations, content of documentations, and difficulties and potential ways of improvement. ${ }^{16}$ We adjusted the topic list several times throughout the study, to ensure data saturation was achieved. Focus group discussions were organized between mid-February and mid-May 2019. A trained and independent moderator facilitated the discussions using the topic list, and an observer (K.D.C.) paid attention to nonverbal communication and group dynamics. During these discussions, which lasted about 45 to 60 minutes, participants explained and deliberated the barriers they experience and their wish list. The discussions were audio-recorded and transcribed verbatim by the observer (K.D.C.).

\section{Data Analysis}

We analyzed the transcripts in duplicate by means of constant comparative technique. ${ }^{17}$ Coding and analyzing took place simultaneously, using NVivo software version 12 Pro (QSR International Pty Ltd). Every 
transcript was coded by 2 researchers independently (E.G.P.M.d.B. and K.D.C.). Inductive content analysis was applied, by using open and subsequently axial coding schemes, leading to main categories and subcategories. ${ }^{16,18,19}$ Contradictions were discussed and resolved by discussion with a third researcher (J.W.L.C.), and the coding scheme was modified several times. Finally, the main categories were discussed among the researchers until consensus was reached, and quotes were selected for each category.

\section{Study Rigor}

We applied several strategies during this study to increase its rigor. First, we achieved investigator triangulation, as researchers with different backgrounds (pharmacy and medicine) were involved, and 2 researchers independently analyzed the data. Combining focus group data with notes on nonverbal communication taken at the focus group discussions and keeping a logbook strengthened our data and methodologic triangulation. In addition, the inclusion of both family physicians and pharmacists, from different cooperatives and having different ages and years of experience, ensured varied data collection. We performed a member check, allowing all participants to check and adapt the transcript, to ensure correct interpretation. Participants were not reimbursed for their time. Finally, the reporting of our study here conforms to the Consolidated Criteria for Reporting Qualitative Research (COREQ) (Supplemental Appendix 1, https://www. AnnFamMed.org/content/18/4/326/suppl/DC1). ${ }^{20}$

\section{Ethics Approval}

Before every focus group discussion, each participant received written information and provided written informed consent. The data of included participants were encoded by numbering, ensuring anonymity and confidentiality. The study was approved by the Medical Ethics Committee of Maastricht University Medical Centre (METC 2019-0969).

\section{RESULTS}

We conducted 4 focus group discussions among 44 participants (34 family physicians, 10 pharmacists). Twenty-six were male. Participants' mean age was 44 years (range $=27$ to 67 years), and they had an average working experience of 14.5 years (range $=0.5$ to 33 years). Data saturation was achieved after 3 focus group discussions.

We identified 3 main themes: (1) magnitude and awareness of the problem of inappropriate antibiotic allergy documentation, (2) origin of this problem, and (3) approaches for addressing it. They are discussed in further detail with selected comments below, with designation of focus group discussion (FGD) number and participant profession, either family physician (FP) or pharmacist $(\mathrm{Ph})$, and number. Figure 1 shows an overview of the identified themes, illustrated with selected quotes. Figure 2 shows the cause-effect relationship regarding the large number of inappropriate antibiotic allergy documentations, with accompanying recommendations for improving this situation.

\section{Magnitude and Awareness of the Problem}

Participants' primary thoughts on inappropriate antibiotic allergy documentation included the lack of clarity of current documentation and the enormous amount of work it would take to evaluate all these unclear and incomplete records. Family physicians and pharmacists mentioned that they are exposed to documented antibiotic allergies every day, because these records are so numerous, and indicated that this problem requires more attention from policymakers involved in developing guidelines and researchers in the field of primary care:

"It (current antibiotic allergy registrations) is nothing but a mess..." (FGD2, FP1)

Despite the fact that family physicians and pharmacists frequently encountered documented antibiotic allergies in daily practice, family physicians concluded that most of them ultimately turn out to be adverse effects instead. They said that real type I reactions are rarely seen in practice:

"I think that in 98 out of 100 patients, you can replace 'allergy' by 'side effect'." (FGD3, FP4)

Some family physicians were aware that inappropriate documentation has consequences, mainly for patients in the hospital. They noted that patients with a documented allergy have limited treatment options in terms of antibiotics, and discussed how this limitation can lead to serious problems in the case of certain infections, especially in life-threatening situations. Many participants seemed to be unaware of the potential negative consequences (antibiotic resistance, increased health care costs, and longer time to recover) when using second-choice antibiotics, however:

"It poses few problems for FPs and pharmacists, there are always alternatives." (FGD2, FP1)

Family physicians and pharmacists indicated that the magnitude of contamination of records with inappropriate and incomplete documentation leads to skepticism regarding its accuracy. They agreed that they most often wonder whether a documented allergy is well grounded: 


\section{Figure 1. Themes regarding the problem of inappropriate antibiotic allergy documentation, with quotes.}

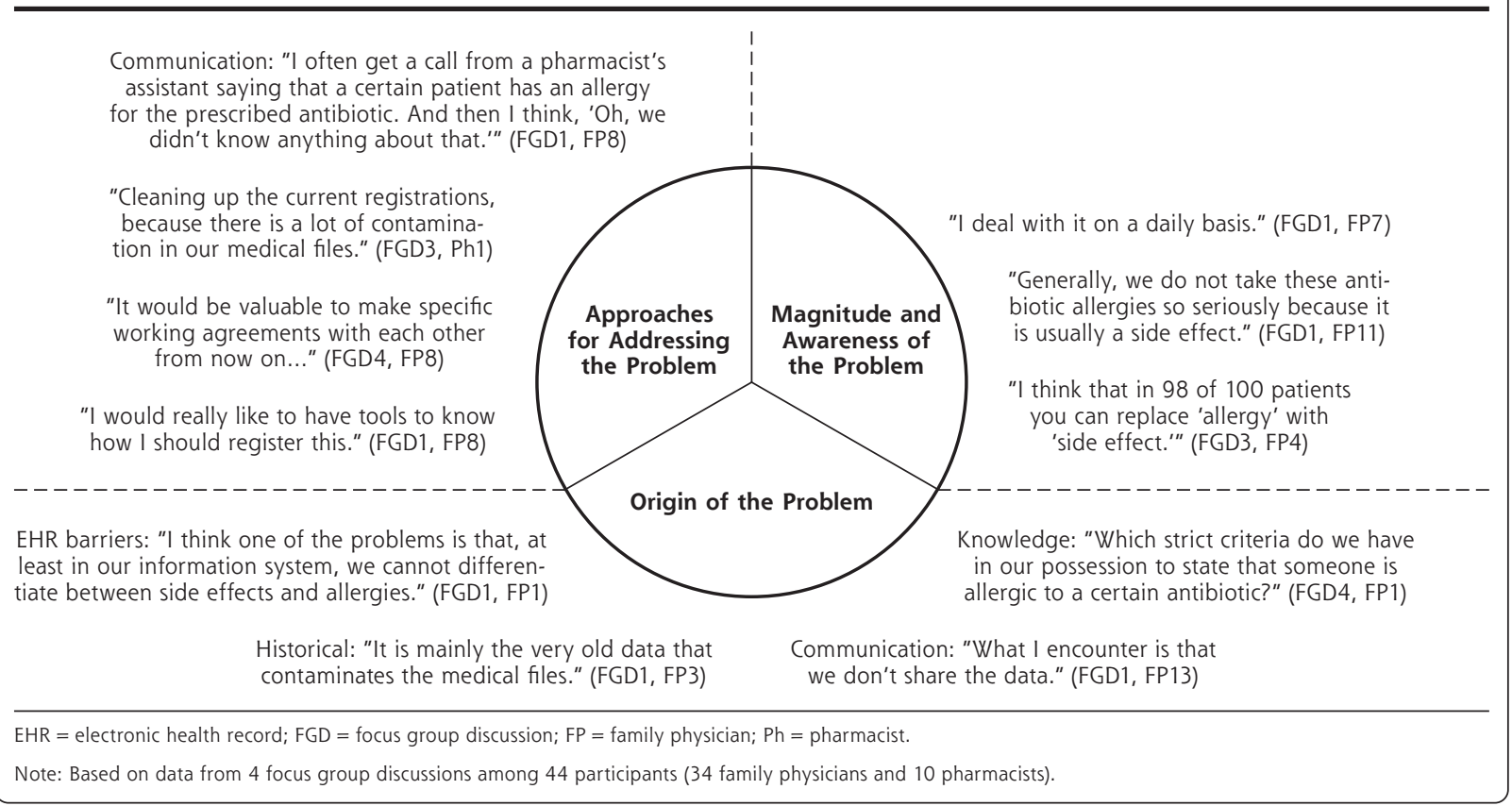

"Generally, we do not take these antibiotic allergies so seriously, because it is usually a side effect." (FGD1, FP11)

Because of incomplete documentation, however, family physicians believed they often had no other option than to prescribe alternative, second-choice antibiotics. Family physicians and pharmacists explained that doubts and fear of a serious allergic reaction, or sometimes even reoccurrence of a serious side effect, were the main reasons to select an alternative antibiotic:

"Better safe than sorry..." (FGD2, FP1)

Some family physicians said that, given their skepticism about accuracy of a documented antibiotic allergy, they went ahead and prescribed the antibiotic anyway, and this practice often turned out well.

Most family physicians and pharmacists were unaware of how to view the number of registered allergies in their electronic health record (EHR) system, resulting in little insight into the prevalence of such documentation in their practice or pharmacy.

\section{Origin of Inappropriate Documentation}

According to family physicians and pharmacists, 5 factors contribute to the numerous inappropriate documentations of allergy: historical factors, EHR barriers, communication regarding documented allergies, responsibility of documentation, and knowledge about antibiotic allergies.

\section{Historical Factors}

Participants perceived that evolution of technology and automation has led to accumulation of inappropriate allergy documentation in medical records. What used to be written manually is now processed in the EHR (information system) in various ways. As a result, documented allergies can be found in numerous places and at numerous levels in medical records. This accumulation could occur, for example, by documenting an allergy in a patient's episode list, by flagging it as a contraindication, or by writing it down at a specific fictional date in the future so that it would always turn up on top of the patient's medical record.

"When I started, we wrote an allergy at the top of 'the green patient chart.' After the automation, there were memos, and now there is a specific field in the electronic medical files intended for intolerances, contraindications, and allergies. As a result, many registrations are mixed up." (FGD4, FP8)

The family physicians and pharmacists generally believed that many adverse effects were documented as allergies in the past because there was insufficient knowledge about antibiotic allergies, and there were fewer options and nuances for documentation in EHRs. In addition to inappropriate documentation of antibiotic allergies, insufficient knowledge about other relevant factors (eg, viral infections that can cause similar symptoms) complicated matters. Family physicians and pharmacists stated there is therefore much uncertainty stemming from incomplete information: 


\section{Figure 2. Cause-effect diagram for inappropriate antibiotic allergy documentation.}

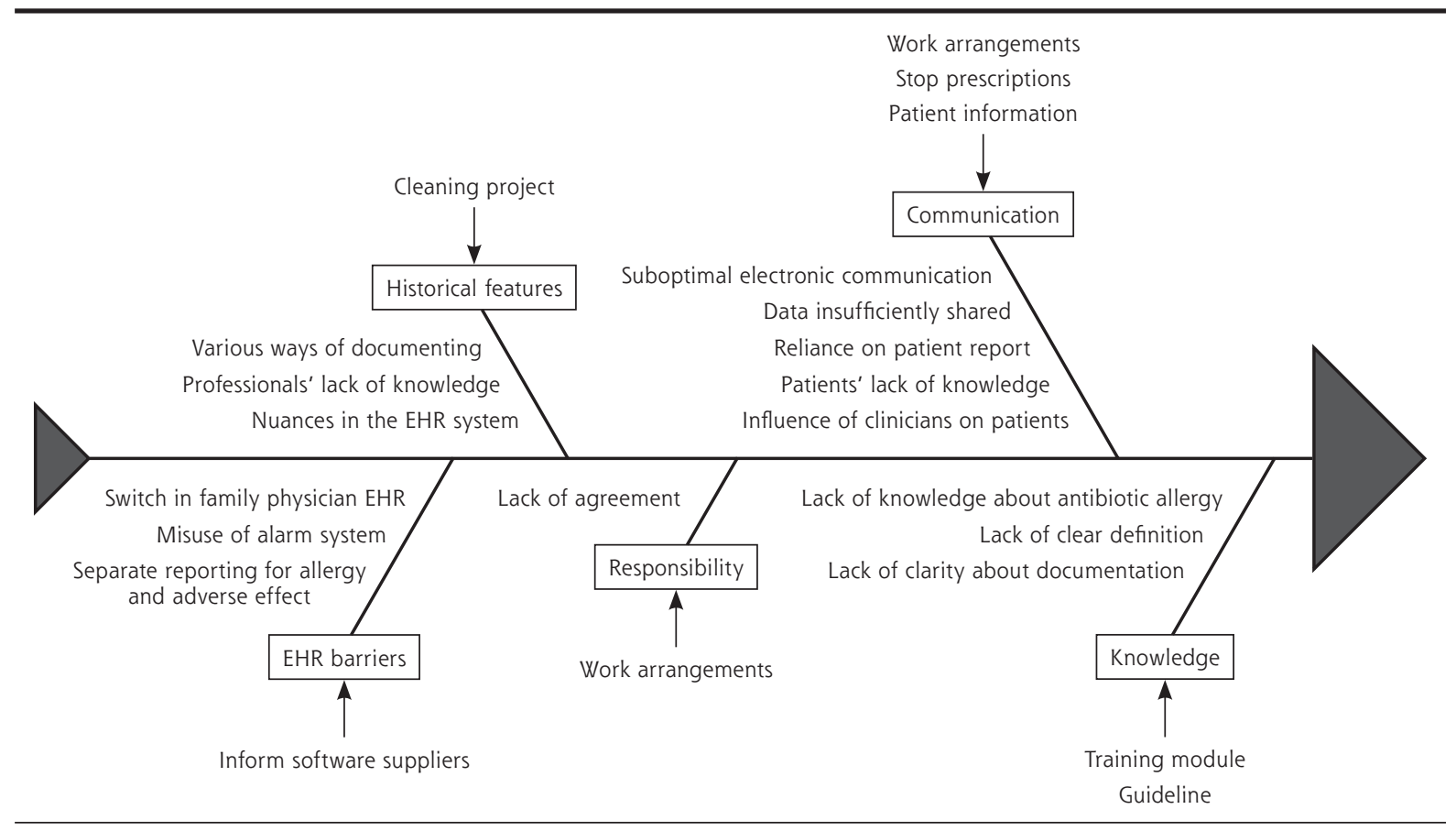

$\mathrm{EHR}=$ electronic health record.

Note: Causes are shown in boxes. Effect (increase in volume of such documentation) is denoted by arrowheads. Recommendations for addressing the problem are shown by peripheral text with arrows.

"It is mainly the very old data that contaminates medical files." (FGD1, FP3)

"In the past, there was no nuance possible, so all side effects were indicated as an allergic reaction." (FGD3, FP5)

\section{EHR Barriers}

One of the major hindering factors identified was the fact that current EHR systems (software packages) do not always lend themselves to efficient and correct allergy documentation. According to the family physicians and pharmacists, it is impossible to distinguish adverse effects from allergies in most of these systems:

"I think one of the problems is that, at least in our information system, we cannot differentiate between side effects and allergies." (FGD1, FP1)

In addition, some pharmacists explained that they occasionally documented an allergy on purpose to misuse the alarm system, in order to block certain drugs in a patient's file. They used this practice when a patient did not want a certain drug or brand, because of adverse effects or costs. Family physicians did not mention doing so, most likely because their EHR system works slightly differently and is not designed to allow avoidance of specific brands of a drug, but also because family physicians believed that providing a specific brand is up to the pharmacist:
"I must admit that I also misuse the system from time to time. The only way I can prevent my assistants from providing a certain drug is by registering an allergy for that drug." (FGD1, Ph1)

\section{Communication Regarding Documentation}

Electronic communication between general practices, pharmacies, and hospitals was noted to be suboptimal. Most participants mentioned a lack of communication about antibiotic allergy documentation between family physicians and pharmacists, although not all of the former were aware of this. Some family physicians experienced insufficient sharing of data. Additionally, they were uncertain how much overlap in documentation is present between the general practice and pharmacy:

"I often get a call from a pharmacist's assistant saying that a certain patient has an allergy for the prescribed antibiotic. And then I think, 'Oh, we didn't know anything about that."' (FGD1, FP8)

Besides communication among health care clinicians, family physicians and pharmacists emphasized that communication with patients is extremely important. Most documented allergies are made based on information from patients, for example, patients reporting to a pharmacist that they have an allergy or new patients writing down an antibiotic allergy on their registration form: 
"I think that many allergies have been registered based on oral information from the patient, over the years." (FGD3, FP9)

It was generally agreed that health care clinicians have a major influence on patients' perceptions as to whether they have an antibiotic allergy. Several family physicians therefore mentioned that clinicians should pay attention to the terminology they use when counseling patients about reactions to an antibiotic:

"If a patient believes that he is allergic to a certain antibiotic because a health care provider told him so, I can sincerely imagine that the next time I want to prescribe that antibiotic the patient refuses to take it." (FGD2, FP1)

Participants believe many patients do not understand the meaning of antibiotic allergy and have no insight into the consequences of a documented allergy. According to them, providing good information to patients and involving patients in the decision as to whether to prescribe a certain antibiotic is extremely important:

"If I think about allergies, I think it is striking that patients don't know what it exactly means." (FGD4, FP5)

\section{Responsibility for Documentation}

In general, there was little agreement about whether a single party is responsible for documenting antibiotic allergies in general practices and pharmacies. Participants did agree that responsibility should lie with either clinicians or pharmacists because they are able to evaluate the symptoms. If assistants to physicians and pharmacists are also allowed to perform this task, they will need clear instructions, participants stated:

"Reporting an allergy is a shared responsibility, both of the patient and the physician. Registration is a responsibility of the physician." (FGD1, FP5)

\section{Knowledge of Antibiotic Allergies}

During the focus group discussions, family physicians and pharmacists admitted that they had insufficient knowledge about antibiotic allergies. They indicated that it is difficult for them to distinguish an allergy from an adverse effect, and said there is a need for a clear definition of the former. Additionally, they asked for more clarity about how to document allergies:

"Which strict criteria do we have in our possession to state that someone is allergic to a certain antibiotic?" (FGD4, FP1)

"I would like to have more knowledge, for example, about the difference between an allergy and a skin rash." (FGD1, Ph4)

\section{Approaches for Improving Documentation}

During the focus group discussions, family physicians and pharmacists proposed several interventions for improving accurate documentation of antibiotic allergy. To improve communication between various health care clinicians, participants suggested establishing working arrangements among local health care professionals, for example, by agreeing to use "stop prescription" alerts, supplemented with the reason the drug was stopped on the prescription. This practice could be help improve communication between family physicians and pharmacists:

"It would be valuable to make specific local working agreements with each other from now on..." (FGD4, FP8)

Furthermore, electronic communication among general practices, pharmacies, and hospitals should be improved to ensure optimal connection of their EHR systems. Family physicians and pharmacists suggested several system adjustments. First, they recommended allowing separate reporting of allergies and adverse effects. Second, they proposed enabling ways of combining the allergy label with a detailed description on the type of reaction the patient had when sending it to other information systems. Finally, they suggested creating a separate notification system, in addition to the existing system for allergies and contraindications, when a patient does not want a certain drug, to avoid misuse of the alarm system for allergies. An additional point they mentioned regarding communication was the importance of counseling patients and shared decision making:

"I think a good explanation and good instruction to patients is important." (FGD4, Ph1)

Some family physicians also indicated that the contamination of medical records should be tackled. They requested a toolkit to clean the enormous number of documented allergies in an efficient way. They proposed that active case finding, for example, based on a printout of the patients with a documented allergy using International Classification of Primary Care (ICPC) codes, could be useful. They did, however, fear the magnitude of work that this undertaking would entail:

"It would be different if you could do active case finding by printing out." (FGD1, FP8)

As mentioned above, participants themselves indicated that their knowledge about antibiotic allergies leaves room for improvement. For example, participants believed they were unable to distinguish between an allergy and an adverse effect. They stated that a clear definition of an antibiotic allergy and a clear overview of the different types of reactions is required. Moreover, they wondered to what level one should document the specifics of an allergy and which reactions have a risk of turning into a serious allergic reaction in the future. They all agreed that a training module could help to answer these questions: 
"What I would like to know is at what level you have to register, by substance name, group, or brand?" (FGD1, FP8)

In addition to the request for specific information, participants indicated that they would like to have a primary care guideline or algorithm concerning the documentation of antibiotic allergies. This guideline should contain a checklist with questions to exclude an allergy and should list characteristics of ideal documentation:

"I would really like to have tools to know how I should register this." (FGD1, FP8)

\section{DISCUSSION}

Family physicians and pharmacists encounter documented antibiotic allergies daily. Participants in our study were aware that such documentation seldom concerned potentially life threatening, type I reactions, and described how the magnitude of contamination of medical records with inappropriate documentation leads to skepticism. Nevertheless, because of incomplete documentation, family physicians feel they often have no other option than to prescribe alternative, second-choice antibiotics. The large-scale consequences of using these broad-spectrum antibiotics were not generally recognized. The enormous amount of inappropriately documented allergies originates, according to our participants, from contamination of medical records in the past, current EHR and communication barriers, little agreement about the responsibility for and way of documenting allergies, and lack of knowledge and facilitating tools. To increase accuracy of future documentation, they suggested forging local working agreements, facilitating better communication between EHR systems and with patients, and improving clinician knowledge through a training module and development of primary care guidelines regarding the documentation process.

To our knowledge, this is the first qualitative study focusing on family physicians' and pharmacists' experiences regarding inappropriate antibiotic allergy documentation and its causes. The primary strength of this study is that it provides an insight into the origin of this extensive problem. Furthermore, the setting is important as most antibiotic allergies are likely documented in primary care, ${ }^{11}$ and the qualitative design enabled investigation of the needs and suggestions of family physicians and pharmacists to improve future documentation. The identified barriers and possible solutions were schematically visualized using a cause-effect diagram (Figure 2). A trained and independent moderator facilitated the discussions by asking open-ended questions, with the intention to reduce the influence of the researchers' opinions. Furthermore, the researcher, data, and methodologic triangulation, and application of the COREQ criteria, ${ }^{20}$ increased study rigor.

Some of the discussed barriers to addressing the problem are related to the Dutch health care and educational systems, which influences transferability of the findings to other countries to a certain extent. A detailed description of the methods and participants should allow others to decide whether the results will apply to their context. Although we carefully considered the chosen methodology, we cannot exclude the possibility that participants gave socially acceptable answers.

Family physicians and pharmacists believe that in most cases, inappropriate documentation of an antibiotic allergy originates from contamination of medical records in the past, EHR and communication barriers, uncertainty about responsibility, and lack of knowledge. These factors have not been described in the literature before. Record contamination and EHR barriers are unanticipated hazards of computerization that need further exploration by policy makers and software developers.

In line with a previous quantitative literature, ${ }^{17}$ we determined that many family physicians and pharmacists found numerous documented antibiotic allergies to be inappropriate. Furthermore, participants mentioned that documentation is incomplete, and they lacked a clear definition of antibiotic allergy. The inconsistent use of terminology regarding antibiotic allergies has been previously described. ${ }^{21}$ Other knowledge gaps that emerged in our study have been discussed in literature; for example, family physicians and pharmacists were seldom aware that the time between intake of an antibiotic and a reaction is rarely noted in medical records, and that an antibiotic allergy is not a permanent condition. ${ }^{22,23}$

Although Wanat et al ${ }^{24}$ had a different research question, focusing on barriers to using allergy services for penicillin allergy testing, their findings are in line with ours. They similarly describe how clinicians are aware that many documented penicillin allergies are inappropriate, but are reluctant to modify them based on their clinical assessment. Both studies indicate that better communication with patients is essential given their insufficient knowledge about the consequences of reporting an antibiotic allergy for themselves and others. ${ }^{24}$

Our study provides insight into the causes of the high number of inappropriate antibiotic allergy documentations with associated potential ways of improvement. Future research should investigate applicability and feasibility of the practical advice given.

To improve the accuracy of future documentation, participants proposed increasing focus on 
communication through practical local working arrangements and development of patient education material. EHR barriers should be addressed with software providers (eg, by modifying systems to report allergies and adverse effects separately and enable easy, reliable communication between systems). A clean-up of current documented allergies is required, although a clear, methodical approach to delabeling inappropriate documentation needs further investigation. Finally, professionals' allergy knowledge can be enhanced using a training module, and documentation can be facilitated by developing a guideline and practical tools such as an algorithm.

In conclusion, to our knowledge, this is the first qualitative study focusing on family physicians' and pharmacists' experiences regarding causes of inappropriate antibiotic allergy documentation. These professionals perceived that antibiotic allergy documentation is seldom accurate, which may contribute to development of antibiotic resistance, increased costs, and decreased patient safety. EHR and communication barriers, and a lack of knowledge and facilitating tools are main causes Practical working arrangements, proper communication between EHR systems, cleaning up old documentation, creating a training module to improve knowledge, and developing tools to relabel inappropriate documentation and to facilitate accurate future documentation are potential ways to improve documentation.

\section{To read or post commentaries in response to this article, see it online at https://www.AnnFamMed.org/content/18/4/326.}

Submitted August 20, 2019; submitted, revised, November 13, 2019; accepted November 27, 2019.

Key words: antibiotic allergy; drug-related side effects and adverse reactions; documentation; inappropriate registration; antibiotic resistance; qualitative research; electronic health records; primary care; practice-based research; health information technology

Funding support: There was no specific funding for this study.

Acknowledgments: We would like to thank Dr Marjorie NelissenVrancken for moderating the focus group discussions and all the family physicians and pharmacists who agreed to participate in this study.

Author contributions: J.W.L.C. and E.G.P.M.d.B. conceived the idea for this study. K.D.C. is the principal investigator and wrote the first version of the manuscript. All authors commented on the first draft and all further revisions of this manuscript.

- Supplemental materials: Available at https://www.AnnFamMed. org/content/18/4/326/suppl/DC1/.

\section{References}

1. Salden OAE, Rockmann H, Verheij TJM, Broekhuizen BDL. Diagnosis of allergy against beta-lactams in primary care: prevalence and diagnostic criteria. Fam Pract. 2015;32(3):257-262.

2. van Dijk SM, Gardarsdottir H, Wassenberg MW, Oosterheert JJ, de Groot MC, Rockmann H. The high impact of penicillin allergy registration in hospitalized patients. JACl: In Practice. 2016;4(5):926-931.
3. Penicillin allergy-getting the label right. Drug Ther Bull. 2017; 55(3):33-36.

4. MacLaughlin EJ, Saseen JJ, Malone DC. Costs of beta-lactam allergies: selection and costs of antibiotics for patients with a reported beta-lactam allergy. Arch Fam Med. 2000;9(8):722-726.

5. Macy E, Contreras R. Health care use and serious infection prevalence associated with penicillin "allergy" in hospitalized patients: a cohort study. JACI: In Practice. 2014;133(3):790-796.

6. Lee $C E$, Zembower TR, Fotis MA, et al. The incidence of antimicrobial allergies in hospitalized patients: implications regarding prescribing patterns and emerging bacterial resistance. Arch Intern Med. 2000;160(18):2819-2822.

7. Joint Task Force on Practice Parameters; American Academy of Allergy, Asthma and Immunology; American College of Allergy, Asthma and Immunology; Joint Council of Allergy, Asthma and Immunology. Drug allergy: an updated practice parameter. Ann Allergy Asthma Immunol. 2010;105(4):259-273.

8. MacFadden DR, LaDelfa A, Leen J, et al. Impact of reported betalactam allergy on inpatient outcomes: a multicenter prospective cohort study. Clin Infect Dis. 2016;63(7):904-910.

9. Irawati L, Hughes JD, Keen NJ, Golledge CL, Joyce AW. Influence of penicillin allergy on antibiotic prescribing patterns and costs. J Pharm Pract Res. 2006;36(4):286-290.

10. Borch JE, Andersen KE, Bindslev-Jensen C. The prevalence of suspected and challenge-verified penicillin allergy in a university hospital population. Basic Clin Pharmacol Toxicol. 2006;98(4):357-362.

11. Su T, Broekhuizen BDL, Verheij TJM, Rockmann H. The impact of penicillin allergy labels on antibiotic and health care use in primary care: a retrospective cohort study. Clin Transl Allergy. 2017;7:18.

12. Lincoln YS, Guba EG. Naturalistic Inquiry. Thousand Oaks, CA: Sage Publications, Inc; 1985.

13. Denning JDVC. Using the focus group in assessing training needs: empowering child welfare workers. Child Welfare. 1993;72(6):569-579.

14. Kitzinger J. Qualitative research. Introducing focus groups. BMJ. 1995;311(7000):299-302.

15. Kitzinger J. The methodology of focus groups: the importance of interaction between research participants. Soc Health Illness. 1994; 16(1):103-121.

16. Bowen G. Grounded theory and sensitizing concepts. Int J Qual Methods. 2006;5(3):12-23.

17. Pope C, Ziebland S, Mays N. Qualitative research in health care. Analysing qualitative data. BMJ. 2000;320(7227):114-116.

18. Corbin JSA. Basics of Qualitative Research. London, United Kingdom: SAGE Publications; 2008.

19. Charmaz K. Constructing Grounded Theory. London, United Kingdom: SAGE Publications; 2006.

20. Tong A, Sainsbury P, Craig J. Consolidated criteria for reporting qualitative research (COREQ): a 32-item checklist for interviews and focus groups. Int J Qual Health Care. 2007;19(6):349-357.

21. Inglis JM, Caughey GE, Smith W, Shakib S. Documentation of penicillin adverse drug reactions in electronic health records: inconsistent use of allergy and intolerance labels. Intern Med J. 2017:47(11): 1292-1297.

22. World Allergy Organization. Drug allergies. http://www. worldallergy.org/education-and-programs/education/allergic-diseaseresource-center/professionals/drug-allergies. Published Jan 2007. Updated 2014. Accessed Aug 2019.

23. Blumenthal KG, Shenoy ES, Hurwitz S, Varughese CA, Hooper DC, Banerji A. Effect of a drug allergy educational program and antibiotic prescribing guideline on inpatient clinical providers' antibiotic prescribing knowledge. J Allergy Clin Immunol Pract. 2014;2(4):407-413.

24. Wanat M, Anthierens S, Butler CC, et al. Patient and primary care physician perceptions of penicillin allergy testing and subsequent use of penicillin-containing antibiotics: a qualitative study. J Allergy Clin Immunol Pract. 2019;7(6):1888-1893.e1. 